\title{
Counterpoint
}

Gary W. Moore*

\section{Mixing studies for lupus anticoagulant: mostly no, sometimes yes}

https://doi.org/10.1515/cclm-2019-1248

Received December 3, 2019; accepted December 4, 2019; previously published online December 24, 2019

Abstract: Mixing tests have long been a mainstay in the lupus anticoagulant (LA) testing armoury of screen, mix and confirm assays. If a sample with an elevated screening test does not evidence inhibition in the mixing test, the search for an LA is halted and a different diagnostic pathway embarked upon. Recent years have seen studies evidencing sometimes high frequencies of falsenegative mixing tests with perhaps sinister implications for missed diagnoses and skewed patient management. Issues such as the dilution effect, between-reagent sensitivity and specificity differences, variability of normal pooled plasma (NPP) quality and suitability and interpretive inconsistencies all contribute to questioning the reliability of mixing tests and their pivotal place in the LA assay hierarchy. The advent of integrated testing, where phospholipid-dependence is demonstrated or excluded prior to any attempt to evidence inhibitory properties with a fallible analytical principle, provides an alternative path to LA detection. In the absence of other causes of elevated clotting times, LA assay screen and confirm discordance is sufficient to secure a laboratory diagnosis of the presence of an LA, leaving the mixing test in a supplementary yet valuable role when further diagnostic discrimination is required.

Keywords: activated partial thromboplastin time; dilute Russell's viper venom time; false negative; lupus anticoagulant; mixing test.

Lupus anticoagulants (LAs) are phospholipid-dependent autoantibodies detected by inference from their behaviour in a medley of coagulation assays, performed in sequence [1]. The first is a screening test employing dilute phospholipid to accentuate the in vitro inhibitory effect

\footnotetext{
*Corresponding author: Dr. Gary W. Moore, Department of Haematology, Specialist Haemostasis Unit, Addenbrooke's Hospital, Hills Road, Cambridge CB2 0QQ, UK, Phone: +44 (0) 1223216746 , E-mail: garymooregwm@hotmail.com
}

of LA antibodies and increase likelihood of detection. An elevated screening test can have numerous causes other than LA, such as factor deficiencies or therapeutic anticoagulation, so reflex assays are initiated to narrow down and pinpoint the nature of any abnormality. By definition, LAs are inhibitors, so an elevated screening test is traditionally and reasonably followed up with a 1:1 mixing test with normal plasma to evidence this property. If the screening test performed on the mixture is also elevated, suggesting an inhibitory process, it is necessary to demonstrate phospholipid dependence to secure a laboratory diagnosis of the presence of an LA. This is normally undertaken by re-capitulating the screening test with a concentrated phospholipid component to swamp or overwhelm any LA present and shorten the clotting time. Antibody heterogeneity means that no single assay will detect all LAs and the medley must be applied to at least two different clotting assays of different principles, commonly dilute Russell's viper venom time (dRVVT) and an LAresponsive activated partial thromboplastin time (APTT). Positivity with just one assay is sufficient to conclude the presence of an LA.

At first sight this appears to be a fairly straightforward diagnostic journey, yet it is predicated on the assumption that the pivotal decision point of an elevated mixing test comprises an infallible analytical design principle. However, as is common with coagulation assays, this is not the case and an understanding of the limitations of mixing tests permits more informed application of the assays and increased diagnostic efficacy. Principal among the limitations is that of the unavoidable dilution effect on the antibody titre, which in some cases is sufficient to make the inhibitory effect of the LA undetectable [1, 2]. Consequently, these false-negative mixing test results preclude performance of a potentially informative and diagnostic confirmatory test. Given that studies on dRVVT and/or APTT mixing tests report falsenegative frequencies in the region of $50 \%$ of patients with LA [2-9], and that subsequent factor assays to identify the implied factor deficiency are almost invariably fruitless, rigid and uncritical adherence to employing the mixing test result in isolation as a determining 
diagnostic crossroad is not entirely logical. Whilst it certainly improves analytical specificity and diagnostic confidence when positive, a false negative potentially robs the patient of nonetheless accurate disorder identification with the attendant potential to adversely affect management decisions. With this in mind, guidance in the British Society for Haematology (BSH) guideline offers a more pragmatic and informed approach to interpreting the LA assay composite [10]. Specifically, it states that "Mixing tests are a criterion for LA and improve the specificity. However, they introduce a dilution factor and may make weak LA samples appear negative. In the absence of any other causes of prolonged clotting times, such samples should be considered positive if the screen and confirmatory tests on undiluted plasma give positive results". The message is to perform all three assays in the medley but interpret in light of other available information in place of restrictive, blinkered reliance on the isolated result from an imperfect assay. This is best achieved by employing an LA-unresponsive APTT in routine coagulation screening to identify or exclude non-LA abnormalities, thereby permitting evaluation of separate testing with dRVVT and LA-responsive APTT largely unencumbered by the possibility of interference from another abnormality [11]. Thus, for a non-anticoagulated patient with normal prothrombin time and LA-unresponsive APTT, accompanied by elevated dRVVT and/or LA-responsive APTT screening test where the confirmatory test indicates phospholipid dependence, a mixing test reveals nothing further. Aside from being diagnostically redundant in such scenarios, there are consequent cost, resource and logistical benefits to omitting mixing tests when analytically appropriate. This is the premise of integrated testing where screen and confirm assays are assayed in parallel in order to make an immediate assessment for phospholipid dependence prior to, or without, performance of mixing tests [1, 9].

It is generally accepted that false negatives due to the dilution effect occur with weaker antibodies [2, 10, 12], and external quality assurance schemes have reported false-negative mixing tests as a common cause of misclassification of weaker LAs [13, 14]. Whilst some would separately debate the existence or clinical relevance of weak LAs, false-negative mixing tests arise for reasons other than dilution alone. Reagent properties impact whether a given LA might fulfil traditional diagnostic criteria, so what might be an apparently weak antibody in one assay can appear more potent/avid in another [15]. Indeed, some LAs unequivocally positive in undiluted plasma for both dRVVT and APTT will manifest in the mixing test of one but not the other [2,9], emphasising that reagent sensitivity to a given antibody impacts successful demonstration of inhibition. Extrapolating this paradigm one step further, an antibody can be negative in one assay but positive only in testing on undiluted plasma with the other, failing to demonstrate inhibition in the mixing test due to low potency and/or low reagent sensitivity. Antibody heterogeneity is also a factor in that some apparently weak LAs, based on a minimal degree of screening test elevation with undiluted plasma, will still achieve mixing test elevation whilst other apparently more potent LAs will not $[5,7]$. Similarly, innate assay specificity for LA impacts the frequency of success of its mixing studies in evidencing inhibition, leading to the suggestion that the traditional algorithm cannot be generalised to all LA assays, even for reagents of the same principle [16].

Even when using reagents with good LA sensitivity and specificity, other issues impact mixing test performance. A variety of formulae have been proposed and employed for interpreting mixing tests and current guidelines recommend either the index of circulating anticoagulant (ICA) calculation, or a mixing test-specific cut-off (MTC) derived from the upper limit of a population distribution $[11,17]$. Once a choice is proffered, it inevitably prompts questions of equivalence or superiority, and recent studies have shown that MTC is more sensitive for detection of LA inhibition than ICA $[7,8]$. The issue is further complicated by a recent report evidencing that relative LA sensitivity of an APTT reagent employed in routine coagulation screening should dictate mixing test strategy [18]. The study employed multiple reagents and showed that ICA has greater specificity for non-LA causes of prolonged clotting times but MTC has greater sensitivity to inhibition, neither operating as a perfect discriminator. Thus, an elevated APTT with an LA-insensitive reagent can be immediately assessed for factor deficiency without performing a mixing test. If factor assays reveal nothing untoward, or LA is suspected clinically, dRVVT and LA-sensitive APTT are initiated and MTC employed to interpret mixing tests. Conversely, if initial APTT screening is undertaken with an LA-sensitive reagent, an elevated result is first assessed with ICA to initiate testing for factor deficiency if normal, whilst an elevated ICA would progress immediately to APTT confirmatory test and dRVVT analysis. Aside from the necessity for the normal pooled plasma (NPP) used in mixing tests to be platelet poor, other aspects of this crucial reagent tend to receive less attention. An underrecognised issue is that clotting times can vary between NPP preparations, so those with shorter clotting times may require a more potent LA to elevate a mixing test sufficient to evidence inhibition, thereby risking more false negatives [19]. 
There are clearly issues with consistency and reliability of mixing tests in LA detection and exclusion. It has been suggested that, as current and previous International Society on Thrombosis and Haemostasis (ISTH) guidelines recommend mixing studies, despite their limitations, they should be considered an essential component of laboratory criteria for LA detection. In fact, all current LA guidelines, from ISTH, BSH and Clinical and Laboratory Standards Institute (CLSI), acknowledge the occurrence of false-negative mixing tests, so it is incongruous for them to be considered an essential diagnostic criterion. The CLSI guideline goes one step further than the BSH recommendation to accommodate the dilution effect in interpretive strategies and re-prioritises test order to screen, confirm and then mix only if it will enhance diagnostic decisionmaking. The point about the CLSI re-prioritisation is that practitioners should be cognisant that there are clinical and analytical situations where mixing tests are valuable and blanket omission from diagnostic testing is inadvisable. More specifically, where the possibility of an alternative or concomitant abnormality exists, mixing tests can make the difference between accurate diagnosis and false-negative or false-positive interpretation. In common with the BSH guideline, the CLSI guideline recommends that omitting the mixing test requires lack of evidence of other causes of elevated clotting times and screen and confirm test discordance in LA assays, but additionally, that the confirmatory test result is below the upper limit of the reference interval. This aids exclusion of interferences specific to the LA assay itself over and above information supplied by the routine coagulation screen, making the confirmatory test result a diagnostic decision point [9, 11, 20]. It is important to note that some LAs are not fully overwhelmed by the higher phospholipid concentration in confirmatory reagents and the confirm result may be above the upper limit of normal despite discordance with the screen result indicating the presence of an LA [1, 9, $10,17]$. In extreme cases, screen and confirm discordance in undiluted plasma appears insignificant and the dilution effect of mixing studies permits the discordance to manifest [21]. Performing mixing tests for both screen and confirm assays improves LA detection when other causes of prolonged clotting times are present, and the LA cofactor effect can only be realised with mixing tests $[1,10,11]$.

Overall, the dilution effect, differences in reagent sensitivity and specificity, variability of NPP quality and suitability and inconsistencies in interpretation conspire to make it undesirable and even dangerous to place mixing tests on such a high pedestal as that of critical diagnostic decision point. Most initial diagnostic testing on patients yet to be anticoagulated does not need mixing tests as they add nothing if the confirmatory test is normal [9], and there is significant risk of high numbers of false negatives if mixing tests are given inordinate precedence in testing order and analytical robustness. The reality is that a onesize-fits-all algorithm cannot be applied to such a complex arena as LA detection and the onus is on diagnostic practitioners to accept and recognise when mixing tests can be safely omitted, and perform them where they will enhance interpretive and diagnostic outcomes.

Author contributions: The author has accepted responsibility for the entire content of this submitted manuscript and approved submission.

Disclaimer: The views expressed in this paper are those of the author, and are not necessarily those of Addenbrooke's Hospital.

Research funding: The author reports consultancy fees from Technoclone and DSM Pentapharm.

Employment or leadership: None declared.

Honorarium: None declared.

Competing interests: The funding organisation(s) played no role in the study design; in the collection, analysis, and interpretation of data; in the writing of the report; or in the decision to submit the report for publication.

\section{References}

1. Moore GW. Current controversies in lupus anticoagulant detection. Antibodies 2016;5:22.

2. Devreese KM. Interpretation of normal plasma mixing studies in the laboratory diagnosis of lupus anticoagulants. Thromb Res 2007;119:369-76.

3. Clyne LP, Yen L, Kritz NS, Breitenstein MG. The lupus anticoagulant. High incidence of 'negative' mixing studies in a human immunodeficiency virus-positive population. Arch Pathol Lab Med 1993;117:595-601.

4. Thom J, Ivey L, Eikelboom J. Normal plasma mixing studies in the laboratory diagnosis of lupus anticoagulant. J Thromb Haemost 2003;1:2689-91.

5. Moore GW, Savidge GF. The dilution effect of equal volume mixing studies compromises confirmation of inhibition by lupus anticoagulants even when mixture specific reference ranges are applied. Thromb Res 2006;118:523-8.

6. Chandrashekar V. Dilute Russell's viper venom and activated partial thromboplastin time in lupus anticoagulant diagnosis: is mixing essential. Blood Coagul Fibrinolysis 2016;27:408-11.

7. Kumano 0, Moore GW. Lupus anticoagulant mixing tests for multiple reagents are more sensitive if interpreted with a mixing test-specific cut-off than index of circulating anticoagulant. Res Pract Thromb Haemost 2017;2:105-13.

8. Depreter B, Devreese KM. Differences in lupus anticoagulant final conclusion through clotting time or Rosner index for mixing test interpretation. Clin Chem Lab Med 2016;54:1511-6. 
9. Moore GW, Maloney JC, de Jager N, Dunsmore CL, Gorman DK, Polgrean RF, et al. Application of different lupus anticoagulant diagnostic algorithms to the same assay data leads to interpretive discrepancies in some samples. Res Pract Thromb Haemost 2017;1:62-8.

10. Keeling D, Mackie IJ, Moore GW, Greer I, Greaves M. British Committee for Standards in Haematology. Guidelines on the investigation and management of antiphospholipid syndrome. Br J Haematol 2012;157:47-58.

11. Clinical and Laboratory Standards Institute (CLSI). Laboratory testing for the lupus anticoagulant: approved guideline; CLSI Document H60-A. Wayne, PA, USA: Clinical and Laboratory Standards Institute, 2014.

12. Male C, Lechner K, Speiser W, Pabinger I. Transient lupus anticoagulants in children: stepwise disappearance of diagnostic features. Thromb Haemost 2000;83:174-5.

13. Dembitzer FR, Ledford Kraemer MR, Meijer P, Peerschke El. Lupus anticoagulant testing: performance and practices by North American clinical laboratories. Am J Clin Pathol 2010;134:764-73.

14. Reber G, Meijer P. In ECAT veritas? Lupus 2012;21:722-4.

15. Le Querrec A, Arnout J, Arnoux D, Borg JY, Caron C, Darnige L, et al. Quantification of lupus anticoagulants in clinical samples using
anti-beta2GP1 and anti-prothrombin monoclonal antibodies. Thromb Haemost 2001;86:584-9.

16. Chantarangkul V, Biguzzi E, Asti D, Palmucci C, Tripodi A. Laboratory diagnostic outcome applying detection criteria recommended by the scientific and standardisation committee of the ISTH on lupus anticoagulant. Thromb Haemost 2013;110:46-52.

17. Pengo V, Tripodi A, Reber G, Rand JH, Ortel TL, Galli M, et al. Update of the guidelines for lupus anticoagulant detection. J Thromb Haemost 2009;7:1737-40.

18. Kumano O, Moore GW. Ruling out lupus anticoagulants with mixing test-specific cutoff assessment and the index of circulating anticoagulant. Res Pract Thromb Haemost 2019;3:695-703.

19. Moore GW. Reference interval mean clotting times should not be used to calculate lupus anticoagulant mixing test ratios unless they match the normal pooled plasma clotting time. Thromb Res 2017;159:16-8.

20. Devreese KM. No more mixing tests required for integrated assay systems in the laboratory diagnosis of lupus anticoagulants? J Thromb Haemost 2010;8:1120-2.

21. Favaloro EJ, Bonar R, Marsden K. Lupus anticoagulant testing sometimes mixing is required: potential for false negatives without mixing studies. Blood Coagul Fibrinolysis 2013;24:673-6. 